\title{
Big Data Applications in Electric Energy Systems
}

\author{
Nazreen Junaidi \\ Department of Electrical and Electronic Engineering \\ Faculty of Engineering, Universiti Malaysia Sarawak \\ 94300 Kota Samarahan, Sarawak, Malaysia
}

\author{
Mohamed Shaaban \\ Department of Electrical and Electronic Engineering \\ Faculty of Engineering, Universiti Malaysia Sarawak \\ 94300 Kota Samarahan, Sarawak, Malaysia
}

\begin{abstract}
Data-driven management of electric energy systems could provide major returns to system operation and control. This paper explores the potential applications of big data analytics in electricity grids. The primary sources of data in electric utilities are first outlined. These include phasor measurement units (PMUs), smart meters, intelligent electronic devices (IEDs), weather data, geographic information system (GIS), and electricity market data. Potential applications, relating to fault analysis, state estimation, security assessment, variable renewable energy, and power market operation are further described.
\end{abstract}

\section{Keywords—big data; energy; smart grid}

\section{INTRODUCTION}

With the prevalence of information and communication technologies (ICT) in the last two decades of the 20th century, conventional power systems were gradually gravitating into smart electricity grids at the turn of the 21 st century. Since the ultimate objective of introducing ICT is to achieve a secure, reliable, resilient, and flexible power grid, a myriad of sensors and intelligent electronic devices (IEDs), for measurement, monitoring, and control, were deployed across the infrastructure of the electric utility enterprise. These sensors and IEDs glean much more data than required for their intended original applications in the power grids.

Big data analytics tools provide unequivocal opportunities to extract these unused data, convert them to information, and combine them in computational models to be used to bolster real-time decision making in the operation of electricity grids [1].

This paper presents an overview of the prospective benefits and applications of big data analytics in power systems. The multisource of energy data are first outlined. Applications that can exploit big data technology within the context of the smart grid are then summarized. Finally, conclusions are drawn.

\section{SOURCES OF DATA IN THE ELECTRIC UTILITY}

\section{A. Phasor Measurement Units (PMUs)}

Energy data in electric utility are produced from several sources. Traditionally, Supervisory Control and Data Acquisition (SCADA) analog system is used in power grids for several decades to generate data for electric utilities with a sampling rate of 2-4 samples per second. However, some particular events encountered in power systems cannot be captured by this limited sampling rate. For instance, transient stability, which manifests the capability of the system to

\footnotetext{
978-1-5090-6664-3/17/\$31.00 C2017 IEEE
}

recover after being subjected to a large disturbance, usually occur in subseconds. Hence, it is difficult to monitor the stability of the transient and its ensuing oscillations in the system with real time measurements of multiple remote terminal units (RTUs) on the grid, with the data gleaned from SCADA. Consequently, Phasor Measurement Units (PMUs) were introduced into the power system, due to their much higher sampling rate of 30-60 samples per second [1]. PMUs are useful tools for monitoring the performance of the system. Direct time-stamped voltage/current magnitudes as well as phase angles are produced by PMUs. These data are synchronized by global positioning system (GPS) timing and are referred to as synchrophasor data [2].

Wide area monitoring systems (WAMS) are comprised of network of PMUs, deployed at selected locations in the power system, and stores data in a data concentrator every 100 milliseconds. Such large amount of new data have never been traditionally observed before. In the US, total PMUs being set set up have reached over 1546 due to the implementation of American Recovery and Reinvestment Act (ARRA), covering approximately $100 \%$ of transmission system of the US by the end of 2015. While in China, 1717 PMUs had been installed by the State Grid and Southern Grid by the end of 2013. [3], [4].

PMU data are not only valuable to derive preventive corrective control actions in response to transient system events, but they also have a good potenial for a plethora of applications such as state estimation, monitoring of voltage stability and control, validation of load model, and system resortion.

\section{B. Smart Meters}

Operational information are essential for the functioning of power distribution networks. Sources of information are such as Advanced Metering Infrastructure (AMI). AMIs establish communications with the loads and provide time stamped system information. AMIs are basically composed of smart meters as well as two-way communicatin networks. The communication networks transmit electrcity consumption data as well as other parameters of the electrical load and supply to the utility. In addition, it can communicate price signals from the utility to the meter to control the load demand [5].

Unlike conventional meters or automatic meter reading (AMR) in which data were recorded once a month, smart meters collect consumption data every 15 minutes. With such meters, the utility can receive information about the meter such as whether the meter had been tampered with or other incidents 